\section{Nanopowder spray for temporary hemostasis in a patient presenting with esophago-carotid fistula after radiation and surgery for a head and neck cancer}

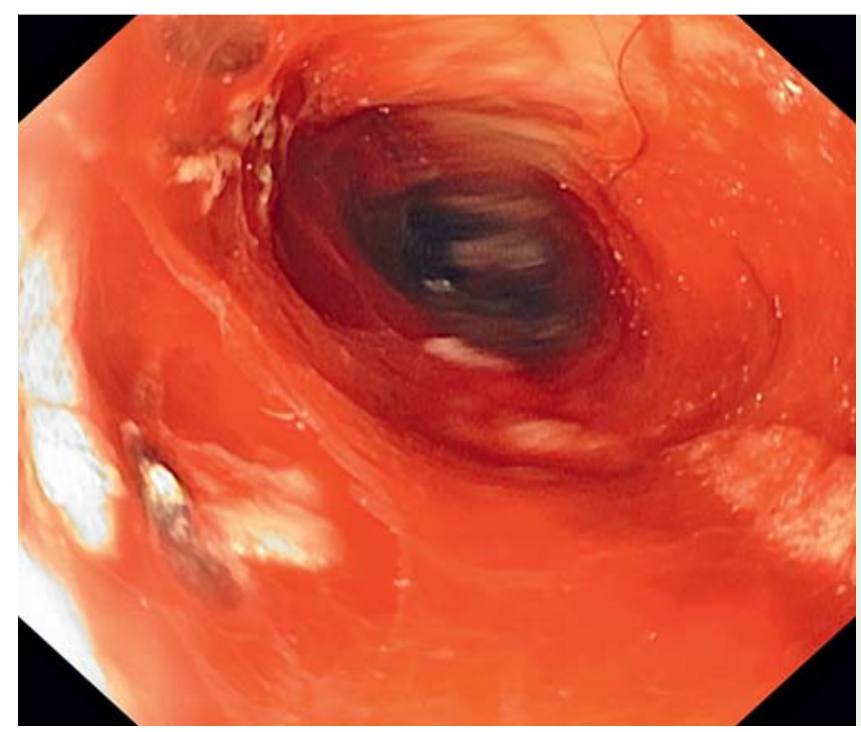

Fig. 1 Patient with esophago-carotid fistula after radiation and surgery for a head and neck cancer. Arterial hemorrhage in the left suture line between the reconstructed neopharynx and the cervical esophagus.

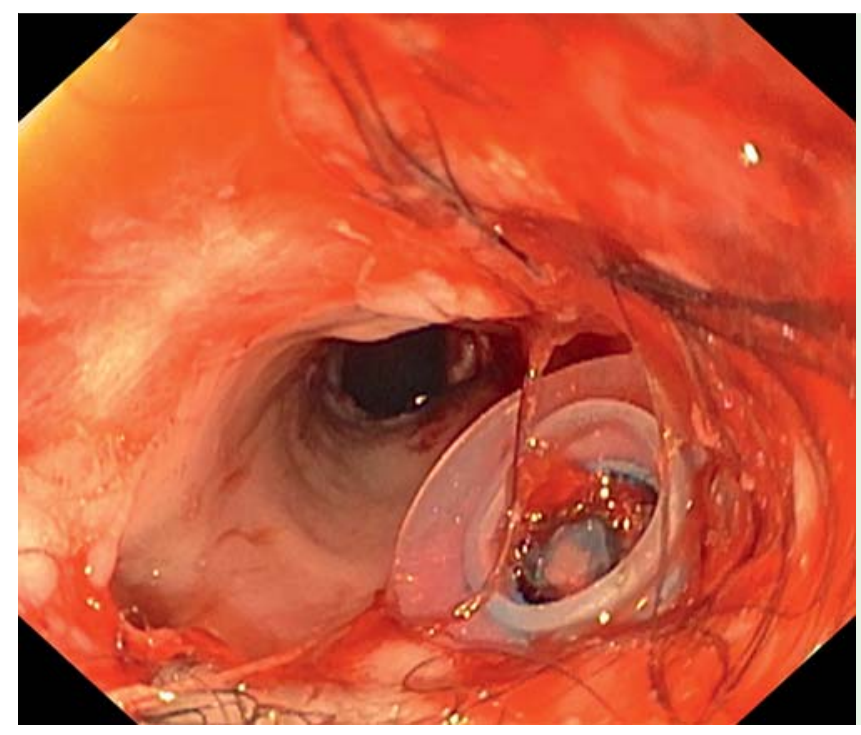

Fig. 2 Pectoralis major flap proximal to a Provox (Atos Medical Horby, Sweden) prothesis (esophagus).

Hemospray (Cook Medical, WinstonSalem, North Carolina, USA), a locally applicable hemostatic agent, was introduced in 2011 for the management of upper gastrointestinal bleeding [1,2]. In contact with moisture, Hemospray becomes cohesive and adhesive, creating a mechanical barrier and effective hemostasis.

A 69-year-old man underwent salvage surgery with total laryngopharyngectomy and reconstruction for a recurrent squa- mous cell carcinoma after primary chemoradiation. After 3 months, the patient was referred urgently to our institution because of acute and massive hematemesis. Hypovolemic hypotension with a positive shock index and a hemoglobin concentration of $2 \mathrm{~g} / \mathrm{L}$ were treated with vasoactive drugs and blood transfusions. The airway was secured with direct intubation through the permanent tracheostomy placed after the laryngectomy.
Endoscopy showed active, pulsating bleeding originating between the pectoralis major flap and cervical esophagus ( $\bullet$ Fig. 1, Fig.2). Because of the massive bleeding, the exact location could not be explored. After the application of Hemospray in the entire neopharynx and proximal esophagus, the bleeding stopped immediately, and the patient was hemodynamically stabilized. Computed tomographic angiography revealed a possible rupture of the left common carotid artery without active bleeding ( $\bullet$ Fig.3a). At 1 hour after the application of Hemospray, massive rebleeding was triggered when the endotracheal tube caused the patient to cough. Definitive hemostasis was achieved with transfemoral placement of a stent graft in the left common carotid artery and internal carotid artery ( Fig. $\mathbf{3}$ b, c).

We present the first case of the effective use of Hemospray for the temporary control of massive bleeding from the upper common carotid artery caused by erosion of the artery. A dehiscence in the suture line between the reconstructed neopharynx and the cervical esophagus with the fistulization of saliva in an irradiated area was the origin of an esophago-carotid fistula with carotid blowout. The immediate application of Hemospray allowed temporary control of the bleeding and stabilization of the patient. Ultimate control was achieved by stent grafting of the carotid artery. Hemospray proved to be effective even in a case of massive and high-pressure bleeding from an esophago-carotid fistula, and the application of Hemospray should be considered in patients with uncontrollable bleeding in the head and neck area.

Endoscopy_UCTN_Code_TTT_1AO_2AD

Competing interests: None

\section{Mikael Sawatzki ${ }^{1}$, Lukas Hechel- hammer ${ }^{2}$, Christa Meyenberger ${ }^{1}$, Ursula Schoenenberger ${ }^{3}$, Sandro J. Stoeckli ${ }^{3}$}

${ }^{1}$ Division of Gastroenterology and Hepatology, Kantonsspital, St. Gallen, Switzerland

2 Department of Radiology, Kantonsspital, St. Gallen, Switzerland

${ }^{3}$ Department of Otorhinolaryngology, Head and Neck Surgery, Kantonsspital, St. Gallen, Switzerland 

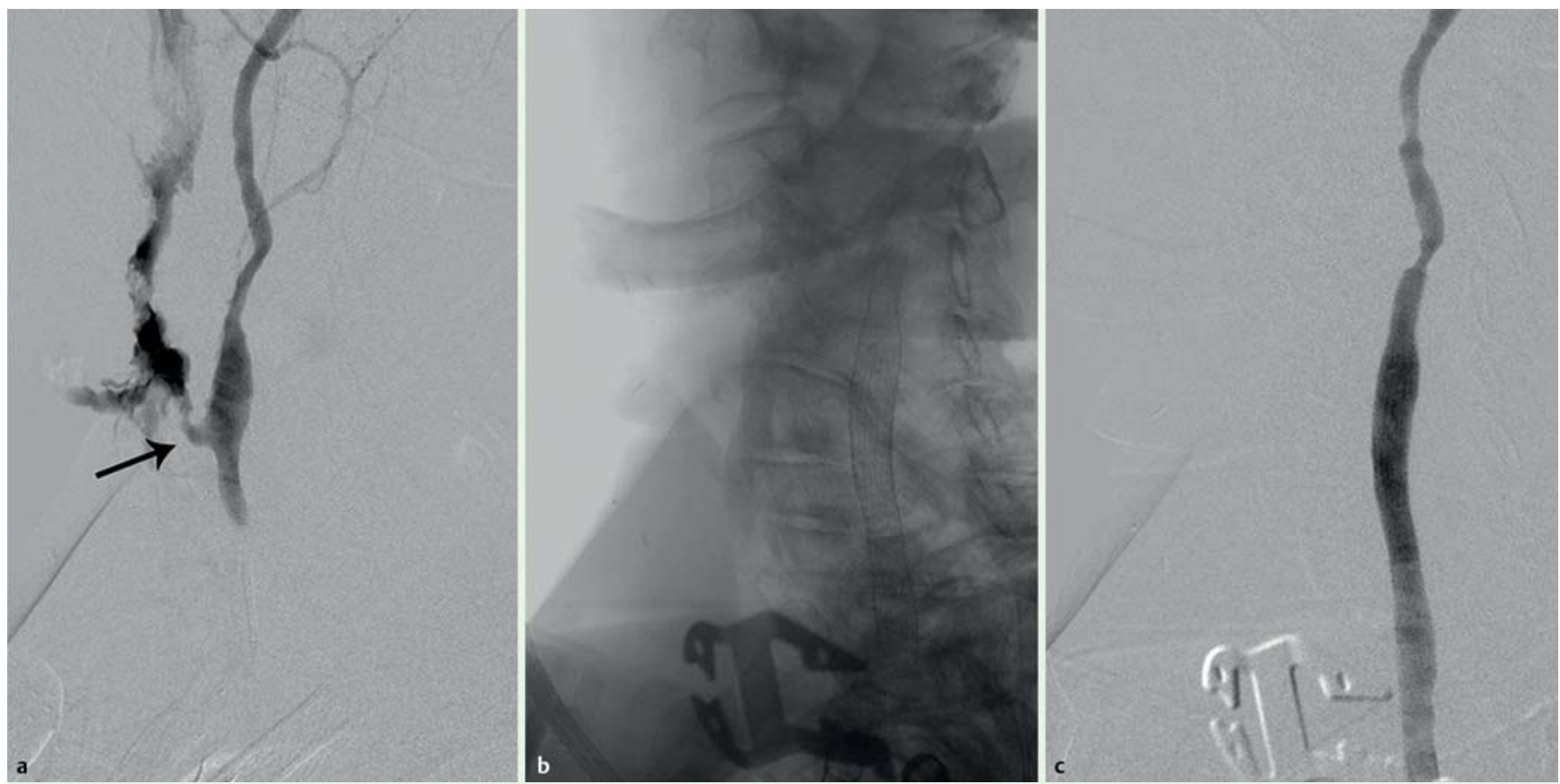

Fig.3 a Digital subtraction angiography showing the rupture site (arrow) of the common carotid artery. b, c Images after stent grafting.

\section{References}

1 Sulz MC, Frei R, Meyenberger $C$ et al. Routine use of Hemospray for gastrointestinal bleeding: prospective two-center experience in Switzerland. Endoscopy 2014; 46: 619-624

2 Sung JJ, Luo $D, W u J C$ et al. Early clinical experience of the safety and effectiveness of Hemospray in achieving hemostasis in patients with acute peptic ulcer bleeding. Endoscopy 2011; 43: 291-295

\section{Bibliography}

DOI http://dx.doi.org/

10.1055/s-0034-1390850

Endoscopy 2014; 46: E644-E645

(c) Georg Thieme Verlag KG

Stuttgart · New York

ISSN 0013-726X
Corresponding author

Mikael Sawatzki, MD

Division of Gastroenterology and Hepatology Kantonsspital

Rorschacherstrasse 195

CH-9007 St. Gallen

Switzerland

Fax: +41714946327

mikael.sawatzki@kssg.ch 